\title{
Electrolocation-communication discharges of the fish Gymnotus carapo L. (Gymnotidae: Gymnotiformes) during behavioral sleep
}

R.M. Stopa and K. Hoshino
Departamento de Ciências Biológicas, Faculdade de Ciências, Universidade Estadual Paulista, Bauru, SP, Brasil

\section{Correspondence \\ R.M. Stopa \\ Departamento de Ciências Biológicas Faculdade de Ciências, UNESP Av. Eng. Luiz Edmundo C. Coube, s/n 17033-360 Bauru, SP \\ Brasil}

Publication supported by FAPESP

Received September 24, 1998 Accepted July 26, 1999

\section{Abstract}

Technical problems have hampered the study of sleep in teleosts. The electrical discharges of Gymnotus carapo L. (Gymnotidae: Gymnotiformes) were monitored to evaluate their ease and reliability as parameters to study sleep. The discharges were detected by electrodes immersed in a glass aquarium and were recorded on a conventional polygraph. G. carapo showed conspicuous signs of behavioral sleep. During these periods, opercular beat rates were counted, electric discharges recorded, and the "sharp discharge increase" (SDI) of the orienting reflex was investigated. All 20 animals monitored maintained electrical discharges during behavioral sleep. The discharge frequencies during sleep $(50.3 \pm 10.4 \mathrm{~Hz})$ were not significantly different from those observed when the fish was awake and inactive $(57.2 \pm 12.1 \mathrm{~Hz})$ (Wilcoxon matched-pairs signed-ranks test, $\mathrm{P}>0.05)$. However, the SDI, which was prevalent in the awake fish, was not observed during periods of behavioral sleep. Additional observations showed that the species had cannibalistic habits. When presented with electrical discharges from a conspecific, the sleeping fish showed an initial decrease or pause in discharge frequency, while the awake fish did not have this response. We conclude that the electrical discharges of G. carapo were not conspicuous indicators of behavioral sleep. Discharges may have been maintained during sleep for sensory purposes, i.e., conspecific detection and avoidance of cannibalistic attacks.

The heuristic value of comparative studies for sleep research is widely recognized, but many animal groups have received little attention. Fish, in particular, have been neglected because of the technical difficulties in obtaining and interpreting conventional electrographic recordings (1). As pointed out by others (1-6), existing studies of sleep in fish are based primarily on behavioral crite-
Key words

- Sleep

- Fish

- Electric discharges

- Cannibalism

- Antipredation mechanism ria, and the universal occurrence of sleep in the group is still questioned $(2,3)$.

Gymnotus carapo L. (Gymnotidae: Gymnotiformes), popularly called "tuvira", is a low voltage electric pulse triggering fish, and may be a potentially valuable species for sleep research. It uses electric organ discharges (EOD) to interact with its environment, e.g., to communicate with conspecif- 
ics and to scan its habitat (7-9). Since reduced interaction with the environment is a marked characteristic of sleep, the electrical discharges of $G$. carapo would be expected to change during a sleep-activity cycle. To investigate this hypothesis, in the present study we determined the occurrence of behavioral sleep in G. carapo and then observed if the state was correlated with changes in EOD. The electrical discharges of this species oscillate between 40 and $100 \mathrm{~Hz}$ and can be measured continuously and easily by a noninvasive technique.

G. carapo individuals of both sexes from the Paraná River basin were used during the study. Size ranged from 50 to $400 \mathrm{~mm}$ standard length. Ten groups of 15 animals were kept in aerated water tanks (1,000-1 capacity) under ambient temperature and light conditions. The fish were fed earthworms daily in amounts corresponding to approximately $10 \mathrm{~g} /$ animal. They were allowed to acclimate for 7 days and then one animal was singled out and transferred to the experimental chamber, an aerated glass aquarium $(250 \times 500 \times 250 \mathrm{~mm})$ surrounded by a visual isolation fence and containing a thermometer. The bottom of the aquarium was filled with a $10-\mathrm{mm}$ deep layer of washed sand. A shelter made with a piece of PVC tubing (24 to $40 \mathrm{~mm}$ in diameter, 50 to $280 \mathrm{~mm}$ in length) was positioned in the sand so that the animal could be observed through a sight hole from outside the visual isolation fence. Electrical cables with 20-mm non-insulated tips were immersed and fixed on the opposite longitudinal walls of the aquarium to transmit and record electric organ discharges via the electromyography channel of an EM polygraph (Electronics for Medicine, Houston, TX, USA).

Preliminary observations were made on 10 animals to evaluate recording quality, baseline discharge frequencies, the effect of temperature, and the response to presentations of some environmental stimuli (light flash, moving shadows, sounds, mechanical vibration of the aquarium, etc). Another 20 fish were observed to determine the occurrence of behavioral sleep. For this purpose, each animal was left undisturbed (except for the placement of worms in the tank) in the experimental aquarium for 7 days. Several times a day, the fish were observed for signs of behavioral sleep, i.e., prolonged immobility in a particular posture, reduced fin movement, and reduction of the frequency of cyclic opercular movements. EOD recordings were carried out when signs of behavioral sleep were apparent and during the orienting reflex test, characterized in the awake animal by a sharp discharge increase (SDI) in response to a plastic rod immersed and stirred in the vicinity of the fish. Near the end of the behavioral sleep recording periods, the aquarium wall was knocked upon to induce locomotion by the fish and to confirm the reversibility of the behavioral state.

Recordings for the first 10 animals at 23$25^{\circ} \mathrm{C}$ showed that inactive, awake fish emitted very regular pulse discharge frequencies ranging from 42 to $68 \mathrm{~Hz}$ (mean $\pm \mathrm{SD}$ of 53.5 $\pm 12.1 \mathrm{~Hz}$ ) (Figure 1). Discharge amplitude changed with animal position in relation to the electrodes and the maximum voltage observed was $1 \mathrm{mV}$. The frequency of pulses increased during swimming, reaching $75.5 \pm$ $16.0 \mathrm{~Hz}$ (mean \pm SD of 15 randomly selected episodes). No relationship was observed between animal size or previous swimming and the frequency of discharge. The mean and standard deviation of the SDI frequency was $71.4 \pm 12.8 \mathrm{~Hz}$. SDI was elicited by rapidly moving shadows and unexpected light changes, observer approximation, knocks on the aquarium wall, or immersion and slow movement of a plastic rod. EOD frequency decreased and increased directly with temperature, but ceased outside the $9-32^{\circ} \mathrm{C}$ range. Near the extremes of this range, the fish lost their natural posture and showed EOD arrest for 3-10 s in response to painful pinching.

There were clear signs of behavioral sleep for G. carapo. Sleep was characterized by a 
motionless posture with the tail down (touching the sand) and the head up (30 degrees with respect to the bottom of the aquarium), reduction in amplitude and frequency of opercular movements, and ventral fin immobility. Plastic rod immersion into the aquarium during these periods did not evoke an SDI, indicating a reduction in the vigilance level. However, the more extreme stimulus of a knock on the aquarium wall woke the fish up as indicated by generalized body movements and undulations of the ventral fins. EOD recordings unexpectedly showed that electrical discharges were maintained during behavioral sleep periods. Discharge frequencies $(50.3 \pm 10.4 \mathrm{~Hz})$ were not significantly different from those recorded during inactive awake periods $(57.2 \pm 12.1 \mathrm{~Hz})$ (Wilcoxon matched-pairs signed-ranks test, $\mathrm{P}>0.05$ ). Amplitude remained constant during sleep and waking periods if the fish did not change its position in the aquarium.

To determine if electrical discharges occurred during sleep via mechanisms independent of those that control other aspects of sleep, behavior and EOD were monitored in anesthetized fish. Thirteen fish were anesthetized for the experiment individually by immersion in an aquarium containing 200 $\mathrm{mg} / \mathrm{l}$ of compound MS-222. Later, they were returned to their original aquarium and monitored to assess the reversibility of anestheticinduced sleep compared with natural sleep. The sequence of behaviors observed was agitated normal swimming, uncoordinated swimming with postural loss (initiated after 10-15 min of latency), and behavioral quiescence with loss of motor responses to pain- ful stimulation (pinching). G. carapo emitted electrical discharges throughout the above behavioral sequence, and then stopped them 5-20 min after quiescence had begun. Because EOD was maintained after behavioral responses had ceased, it is likely to be controlled independently. EOD was recovered after 5-10 min in the original aquarium, concomitantly with postural and swimming normalization.

Interactions with conspecifics were investigated in order to determine the functional role played by the sleep EOD of $G$. carapo. Small size class G. carapo (50-90 $\mathrm{mm}$ ) were introduced into an aquarium with a larger resident animal, which was awake and had been starved for two days (the resident fish was at least $30 \mathrm{~mm}$ longer than the intruder fish). A brief period of attentive behavior of the resident animal was observed, followed by a rapid exit from the PVC shelter, pursuit and capture of the intruder fish, and immediate cannibalism (within 2 to 5 min). Alternatively, in two cases (15.4\%), the smaller fish was killed within the first 5 $\mathrm{min}$, but ingestion was delayed for up to $5 \mathrm{~h}$. The ingestion latencies were shown to increase up to $24 \mathrm{~h}$ if a second or more intruders were introduced (16 observations).

These observations suggest the possibility that $G$. carapo uses its EOD during sleep to detect conspecifics and to avoid cannibalistic attacks. This hypothesis was investigated by transmitting the EOD of a stimulus fish (SF) to a sleeping fish whose EOD was monitored (response fish; RF). SF discharges were transmitted to the RF aquarium by flipping a switch interposed in the cables

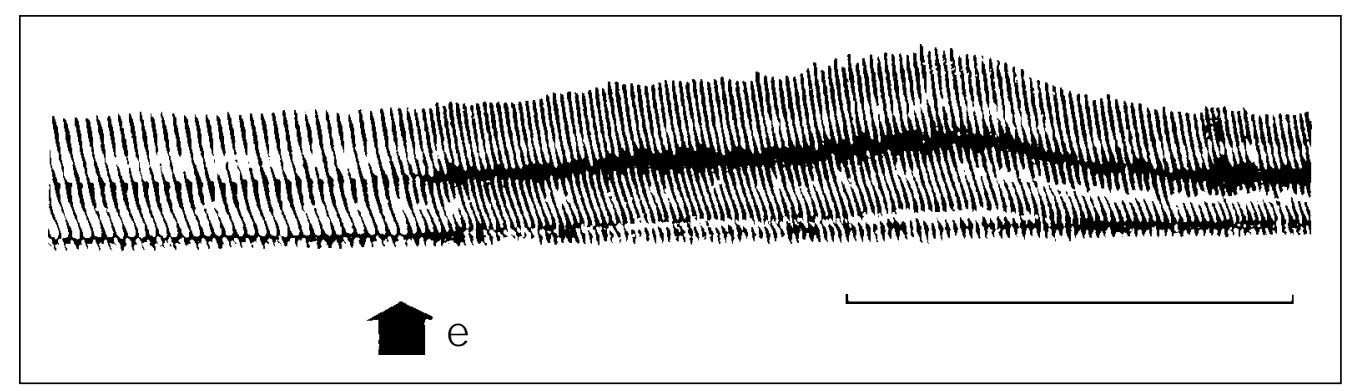

Figure 1 - Electric organ discharge of Gymnotus carapo, used for social communication and electrolocation. A segment of a recording made at rest and after glass rod presentation (arrow) in the animal's vicinity is illustrated. Time calibration: $1 \mathrm{~s}$. e, Stimulus presentation. 
connecting the aquaria. Stimulation lasted about 30-60 s and, in order to compare the response, it was repeated after at least $3 \mathrm{~min}$ if the RF showed signs of being awake. The electrical discharges of 10 fish classified as small (100 to $200 \mathrm{~mm}$ standard length), 10 as intermediate $(210$ to $300 \mathrm{~mm}$ ), and 10 as large (310 to $400 \mathrm{~mm}$ ) were monitored individually on different days during EOD transmissions of small, intermediate, and large stimulus fishes. A total of 90 recordings were made when animals were asleep and 90 when they were awake.

Independent of SF or RF size, 82 (91.1\%) of the tests conducted on sleeping animals showed a discharge frequency reduction or silence during the first $3 \mathrm{~s}$ of SF discharge presentation (Figure 2A). This response was significantly more common than either discharge frequency increases or superpositions (SF and RF simultaneously emitting electrical discharges), which occurred with the other 8 fish (8.9\%) (chi-square $=134.8$, d.f. $=2, \mathrm{P}<0.05$ ). In contrast, $72.2 \%$ of the awake focused fishes displayed discharge frequency increases or superpositions in response to the SF transmissions (Figure 2B). Another $18.9 \%$ of the awake fish showed discharge frequency decreases, and $8.9 \%$ displayed no observable change. A chi-square test showed that increases and superpositions were more frequent than the other responses for awake fish (chi-square $=62.6$, d.f. $=2, \mathrm{P}<0.05$ ). None of the SF-RF size combinations were associated with a particular response during the initial phase of the EOD recordings. Following the first $3 \mathrm{~s}$, the focused fishes showed a variety of EOD patterns. Some were silent for only a brief period and then resumed their EOD, while others remained silent during and for $30 \mathrm{~s}$ or more after the end of SF discharge transmission. Some appeared to be competing with the SF transmission. These fish emitted alternate discharge bursts or simultaneously superimposed their discharges on those of the SF. These observations were not the focus of this study, but it was interesting to note that six of the focused fishes attacked the tips of the transmission cables.

The data obtained indicate that G. carapo does sleep. The species showed a particular sleep posture, relative immobility, increased sensory thresholds, and reversibility of the state, which are the four widely accepted behavioral criteria for sleep (10). Consequently, this freshwater fish can be added to the category of other species in which behavioral sleep has already been demonstrated $(1-6,10)$.

The sustained EOD observed during behavioral sleep in G. carapo may suggest that
Figure 2 - Initial response of Gymnotus carapo to conspecific discharges presented during sleep (A) and wakefulness (B). Segments of polygraphic recordings for the same animal are presented. The stimulus started at the arrows and lasted $30 \mathrm{~s}$. Note the brief interruption of discharge in A, when discharges of the stimulus fish were recorded (low-voltage pulses), and subsequent overlapping discharges (probably competition). Such discharge arrest was not observed when the animal was awake. Time calibration: $1 \mathrm{~s}$. F, Onset of discharge presentation.

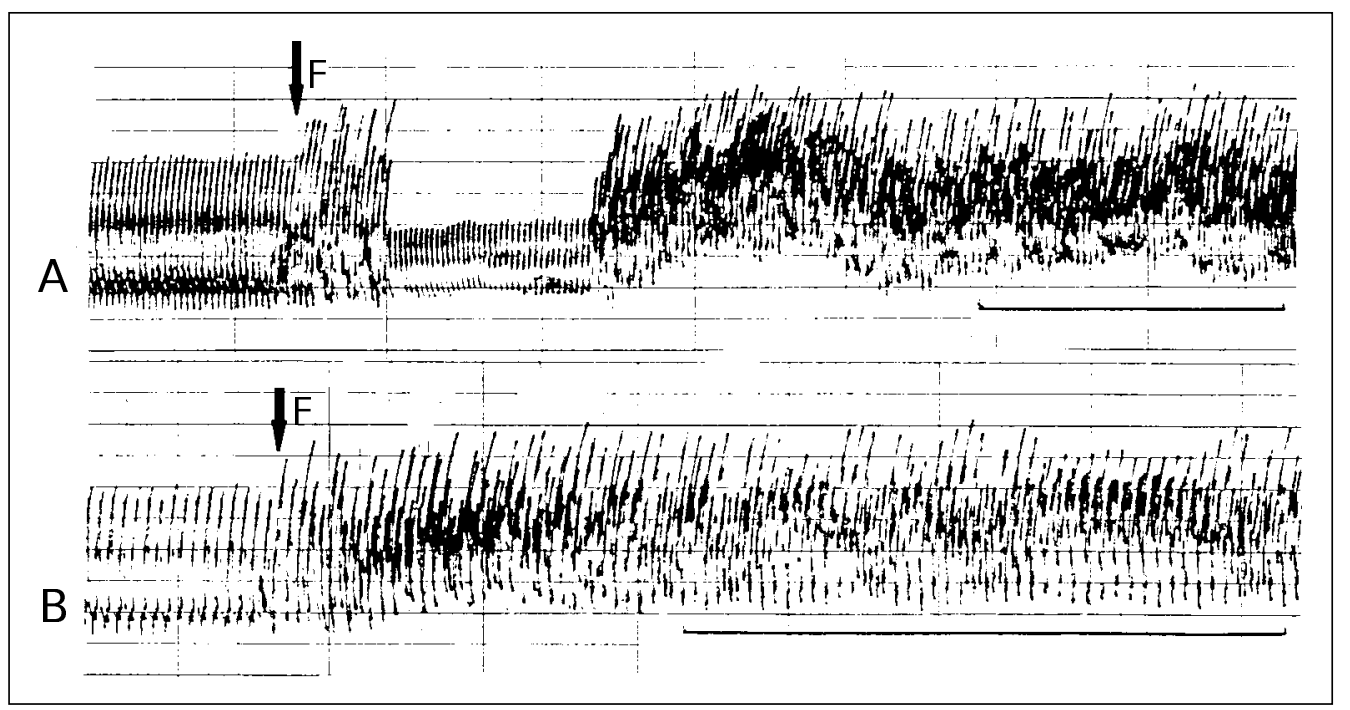


the periods considered as sleep, according to behavioral criteria, are not true sleep. Instead, the periods may correspond to the acrophases of rest periods during the circadian rest-activity cycle. This seems unlikely, however, because electrical discharges continued even after the fish lost their natural posture during pharmacologically induced sleep. In other words, the mechanisms controlling EOD may be largely independent of those controlling behavioral sleep. The reduction or suppression of electrical discharges in the RF, observed when the EOD of an SF were presented during behavioral sleep, seems to be strong evidence for the occurrence of significant changes in central nervous system activity. A similar reduction or silence of EOD in G. carapo, elicited by sensory stimulation, was also reported during light anesthesia (a state in which there are changes in central nervous activity) (Correa SM and Hoffman A, personal communication).

Reduction in cerebral serotonin level (11) and sleep potentiation by vasoactive intestinal peptide (2) corroborate the assumption that behavioral sleep in fish is determined by a specific mechanism that occurs in homeotherms. In addition, maintenance of nonvegetative activities during sleep, like swimming in some fish species and aquatic mammals, is well known. Maintaining a sensory channel active during sleep may also be adaptive. For example, the lowering of the auditory stimulus threshold to awake a rat if the sound is conditioned to a signaling electric shock (12). Maintenance of EOD during sleep may have adaptive significance for $G$. carapo as well, by allowing them to detect conspecific cannibals. Contrary to what was expected, the use of EOD to assess the occurrence of sleep was not possible with the methods used in this study. However, the existence of small, undetectable, differences in EOD during sleep should not be ruled out.

Temporary suppression of visceral activ- ity for the purpose of concealment by predators or prey is a well-known adaptive mechanism in aquatic animals and has been demonstrated mainly in the cardiorespiratory activity of decapod crustaceans $(13,14)$. In fish, however, concealment via cardiorespiratory arrest is not a viable strategy, because suspension of heart and opercular beats can last only a few seconds (15). It is instead an anticipatory protective reflex before the explosive muscular activity of a flight response (15). In contrast, the arrest observed in the electrical discharges of $G$. carapo was lengthy for some fish, suggesting its use for concealment. Considering the cannibalistic habits of the species, lengthy EOD suspension may be considered as part of an antipredation mechanism. In this scenario, the brief initial "silence" period, observed primarily in sleeping fishes, is elicited as part of the startle response and then used to analyze exogenous electrical discharges without interference from their own EOD. The information obtained by the fish during the "silence" period may determine its decision to maintain EOD suppression or resume its electrical discharges. Important points for future investigation include whether heart beats are concomitantly arrested during the periods of EOD suspension, and whether $G$. carapo may detect cardiac electrical activity of conspecifics.

Assuming that the brief "silences" were part of the startle-orienting reflex, it may be possible to explain why a significantly greater number of sleeping G. carapo displayed a reduction or silence of discharge frequencies in response to EOD transmissions from conspecifics. Cardiac arrest induced by startling stimuli has been observed in dominant tilapias (Oreochromis niloticus, Cichlidae: Perciformes), which, because of their dominance, are able to rest. Submissive tilapias, which remain startled almost all the time, do not show the cardiac arrest response (16). This suggests that defensive concealment may be more effective for resting or sleeping 
fishes. For G. carapo, the immediate cessation of electrical discharges avoids or retards its detection, granting sufficient time for identification of exogenous stimuli and initiation of other defensive responses.

Although EOD were not useful for detecting sleep, the present study demonstrated that behavioral sleep occurs in G. carapo. This finding adds to the diversity of taxonomic fish groups in which sleep has been described. Reports of fish species which do not sleep seem to be based, at least partially, on the misinterpretation of observations about species which swim during sleep for passive (ram) ventilation of the gills or move their fins to make postural corrections. The universal occurrence of sleep in fishes seems likely since it has been reported in invertebrates as well $(17,18)$. Seep compensation after a period of deprivation ("rebound sleep") $(19,20)$ indicates the importance of this physiological state among numerous and distantly related animal taxa.

\section{References}

1. PeyrethonJ \& Dusan-Peyrethon D (1967). Étude polygraphique du cicle veillesommeil d'un téleosten (Tinca tinca). Comptes Rendus des Séances de la Société de Biologie, 161: 2533-2537.

2. Campbell SS \& Tobler I (1984). Animal sleep: a review of sleep duration across phylogeny. Neuroscience and Biobehavioral Reviews, 8: 269-300.

3. Lenke R (1988). Hormonal control of sleep-appetitive behaviour and diurnal activity rhythms of cleaner wrasse Labroides dimidiatus (Labridae, Teleostei). Behavioural Brain Research, 27: 73-85.

4. Karmanova IG \& Lazarev SG (1979). Stages of sleep evolution (facts and hypothesis). Waking and Sleeping, 3: 506510.

5. Shapiro CM, Woolf CJ \& Borsook D (1981). Sleep ontogeny in fish. In: Laming PR (Editor), Brain Mechanisms of Behavior in Lower Vertebrates. Cambridge University Press, Cambridge.

6. Melesio-Nolasco $S \&$ Ayala-Guerrero $\mathrm{F}$ (1983). Filogenía del sueño en peces y anfibios. Boletín de Estudios Medicos y Biologicos, 32 (Suppl 1): 101-107.

7. Correa SSAL (1995). Conexões intrínsecas e extrínsecas da região dorsocentral do telencéfalo em Gymnotus carapo (Teleostei: Gmonotoidei). Avaliação de sua participação nos processos de alerta e habituação. Master's thesis, Universidade de São Paulo, Ribeirão Preto, SP.

8. Lissmann $\mathrm{H}$ (1958). On the function and evolution of electric organs in fish. J ournal of Experimental Biology, 35: 156-191.

9. Hopkins CD (1988). Neuroethology of electric communication. Annual Review of Neuroscience, 11: 497-535.

10. Hartse KM (1994). Sleep in insects and nonmammalian vertebrates. In: Kryger MH, Roth T \& Dement WC (Editors), Principles and Practice of Sleep Medicine. WB Saunders, Philadelphia.

11. Fingerman SW (1976). Circadian rhythms of brain 5-hydroxy-tryptamine and swimming activity in the teleost Fundulus grandis. Comparative Biochemistry and Physiology, 54C: 49-53.

12. Trigona MC, Ciancia F \& Bloch V (1968). La profondeur du sommeil paradoxal chez le chat: seuils de stimulation réticulaire et détection des stimuli sonores significants ou non. J ournal de Physiologie, 60: 559560.

13. MCMahon BR \& Wilkens JL (1972). Simultaneous apnea and bradycardia in the lobster Homarus americanus. Canadian J ournal of Zoology, 50: 165-170.

14. Cuadras J (1981). Behavioral determinants of severe cardiac inhibition. Physiological Psychology, 9: 384-392.

15. Oliveira PC \& Hoshino K (1989). Heart rate in fishes during interspecific interactions. Boletim de Fisiologia Animal, 13: 49-54.

16. Stopa RM (1992). Paradas cardíacas da reação de sobressalto em tilápias (Oreochromis niloticus) dominantes e submissas. Master's thesis, Universidade Estadual Paulista, Botucatu, SP.

17. Arechiga MD (1994). Do crustaceans sleep? Newsletter of the World Federation of Sleep Research Societies, 4: 7-9.

18. Tobler I (1994). Evidence for sleep in the scorpion and cockroach. Newsletter of the World Federation of Sleep Research Societies, 4: 9-11.

19. Tobler I \& Bórbely AA (1985). Effect of rest deprivation on motor activity of fish. J ournal of Comparative Physiology, 157: 817-822.

20. Inoue S (1994). Behavioral versus telencephalic sleep. Newsletter of the World Federation of Sleep Research Societies, 4: 11-12. 